\title{
Serpientes de la Gran Área Metropolitana (GAM) de Costa Rica
}

\author{
Sergio Gabriel Quesada-Acuña \\ Laboratorio de Ecología Urbana, Vicerrectoría de Investigación, Universidad Estatal a Distancia (UNED), 2050 San José, Costa Rica; \\ sgbiotropic@gmail.com
}

Recibido 18-X-2017 • Corregido 14-XI-2017 • Aceptado 11-XII-2017

\begin{abstract}
Snakes from the Greater Metropolitan Area of Costa Rica. The GAM is a small geographic area that concentrates most of the Costa Rican population, but it that does not contain all the biodiversity that makes the country famous. Here I review information on snakes that coexist with humans in the area. From a total of 142 species, 73 occur in the GAM: 21 species dwell in leaf litter (harmless), two are semi-aquatic species (harmless), 16 tree species (13 harmless) and 34 terrestrial species ( 26 harmless). Only 11 species pose a real danger to human health but, we can coexist with them in peace.
\end{abstract}

Key words: City, Urban ecology, description, environmental education, snakes.
RESUMEN: La GAM es un área geográfica pequeña que concentra la mayor parte de la población costarricense, pero que no contiene toda la biodiversidad que hace famoso al país. Aquí reviso información sobre serpientes que conviven con humanos en el área. De un total de 142 especies, 73 ocurren en la GAM: 21 especies habitan en hojarasca (inofensivas), dos son especies semi-acuáticas (inofensivas), 16 especies arbóreas (13 inofensivas) y 34 especies terrestres (26 inofensivas). Solo 11 especies representan un peligro real para la salud humana pero, podemos coexistir con ellas en paz.

Palabras clave: Ciudad, ecología urbana, descripción, educación ambiental, serpientes.
Costa Rica, un pequeño país centroamericano de tan sólo 51 100km² (MIDEPLAN, 2014), es reconocido internacionalmente por mantener la cobertura natural de sus ecosistemas en el $55.6 \%$ de su territorio continental, lo cual le permite albergar un $5 \%$ de la biodiversidad mundial conocida (SINAC, 2014). Esta realidad costarricense, producto de la interrelación histórica de múltiples factores climáticos, biogeográficos, políticos y socioculturales, posiblemente refleje un aspecto positivo de concentrar el $60 \%$ de la población (MIDEPLAN, 2014) en un área de aproximadamente $2000 \mathrm{~km}^{2}$ Ilamada Gran Área Metropolitana (GAM) (Fig. 1).

La GAM de Costa Rica es un núcleo urbano (residencial e industrial), que permanece creciendo rápida y desordenadamente hacia los cordones montañosos que lo rodean (Barrientos, 2010; INVU, 2011), generando cada vez más presión sobre los recursos naturales y la biodiversidad de la zona (Barrientos, 2010; SINAC, 2014). La GAM abarca 31 cantones de cuatro provincias (Apéndice
1) y se espera que al año 2030 su desarrollo inmobiliario sea aún mayor (INVU, 2011; MIDEPLAN, 2014), de manera que curiosamente, la mayor parte de la población costarricense habita (o habitará) en un área geográfica reducida, que no alberga la totalidad de la biodiversidad que hace famoso al país (SINAC, 2014).

Dado que en la GAM reside la mayoría de la población, pero sólo un porcentaje de la biodiversidad (independientemente si es amada o temida); todo esfuerzo de educación ambiental a las poblaciones urbanas haría bien en considerar la distribución de las especies para establecer prioridades que hagan más "aplicable" el conocimiento adquirido (Barrientos \& Monge-Nájera, 2010). Por esta razón, y considerando la poca empatía que existe entre los seres humanos y las serpientes (Prokop, Özel \& Usak, 2009; Nonga \& Haruna, 2015; Torkar, 2015; Pinheiro, Mota \& Borges-Nojosa, 2016), en este trabajo busco educar y evitar la muerte innecesaria de muchas serpientes urbanas. 


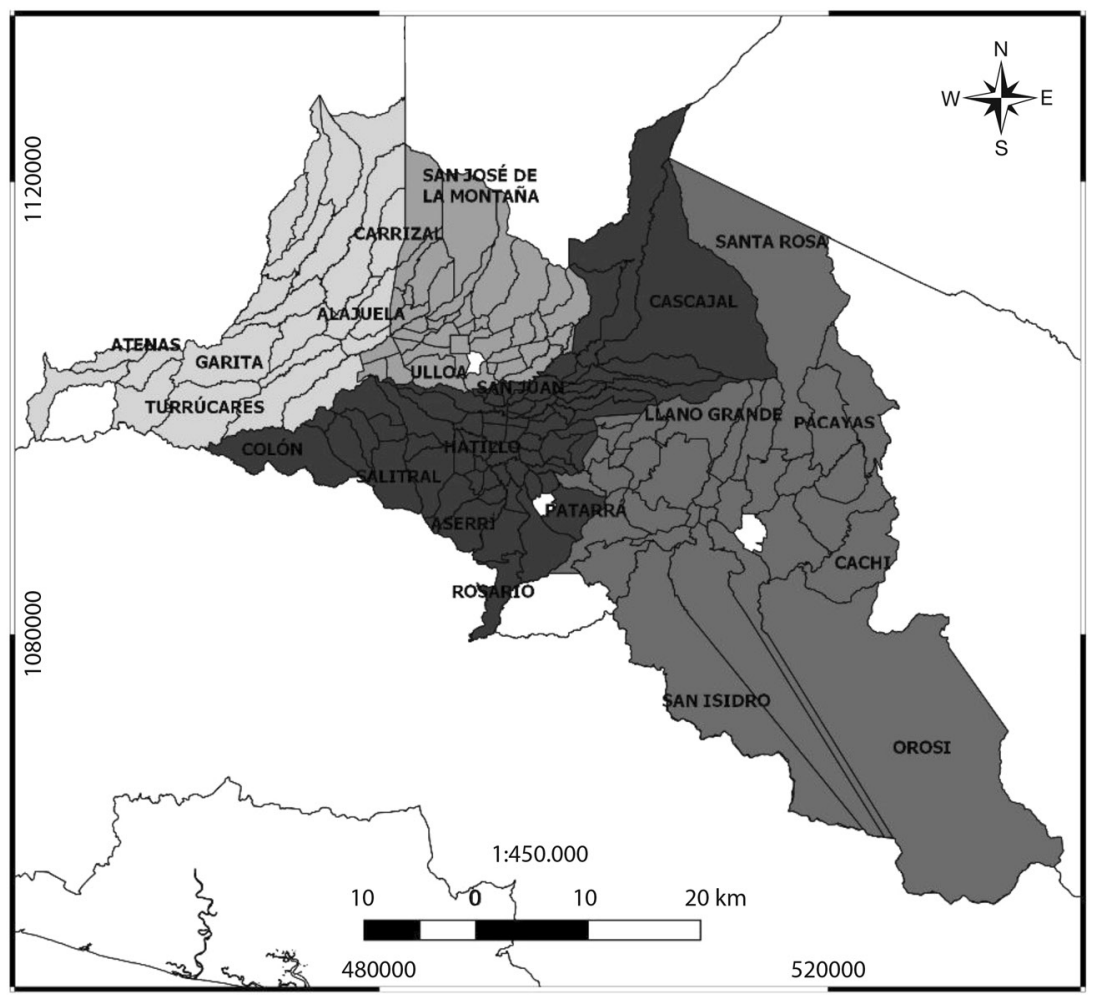

Gran Área

Metropolitana

(GAM), Costa Rica

Leyenda

Distritos urbanos por provincia

$\square$ Alajuela

$\square$ Cartago

$\square$ Heredia

San José

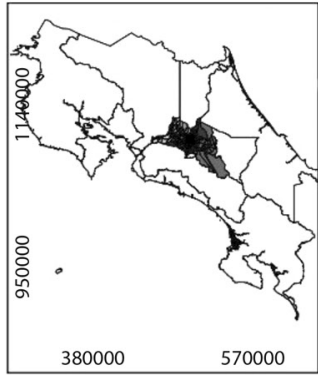

Proyección CRTM05

Datum WGS84

Fuente: www.snit.cr.go.cr,

basado en INVU (2011)

Elaborado por:

Sergio G. Quesada-Acuña

San José, Agosto 2017

Fig. 1. Delimitación de la Gran Área Metropolitana (GAM) de Costa Rica, según el Instituto Nacional de Vivienda y Urbanismo (INVU). Puede ver la lista completa de cantones y distritos en el apéndice 1.

\section{¿Cuánto se conoce sobre las serpientes costarricenses?}

El estudio de las serpientes en Costa Rica es una disciplina que inició aproximadamente en el año 1854 (Savage, 2002), cuando los alemanes Alexander von Frantzius y Carl Hoffmann, junto a sus aprendices costarricenses José Cástulo Zeledón, Juan J. Cooper, José Fidel Tristán y Anastasio Alfaro, empezaron a recorrer el país para recolectar e inventariar todo tipo de especies (Savage, 2002). De aquellas primeras exploraciones (casi aficionadas), ya han pasado más de 160 años en los que decenas de renombrados investigadores (nacionales y extranjeros), han investigado en el campo y en laboratorio para enriquecer continuamente el conocimiento sobre la historia natural, la peligrosidad y la distribución geográfica de las serpientes de Costa Rica (Savage, 2002).

El conocimiento científico sobre serpientes costarricenses es amplio, se encuentra a disposición del público general y ha sido la fuente primaria de información para este documento, pues varios especialistas han recopilado el trabajo de sus vidas en libros ilustrados de cientos de páginas, que se convierten en verdaderos tesoros del conocimiento y álbumes de la biodiversidad costarricense. Como ejemplos, se pueden recomendar los trabajos casi "enciclopédicos" del estadounidense Jay Savage (2002) y del costarricense Alejandro Solórzano (2004); pero también existen "guías de campo" bastante completas del holandés Twan Leenders (2001), del costarricense Federico Muñoz Chacón junto al estadounidense Richard Dennis Johnston (2013), del estadounidense Gregory McConnell (2013), entre otras. Al respecto es importante aclarar que decenas de especialistas no son mencionados en este apartado simplemente porque a la fecha no han publicado grandes libros recopilatorios; aunque definitivamente, sus investigaciones fueron la base para todos los libros mencionados (Savage, 2002; Solórzano, 2004; Muñoz \& Johnston, 2013).

Al revisar exhaustivamente la bibliografía disponible, es fácil notar que el número de especies de serpientes que habitan en Costa Rica varía constantemente por la aplicación de novedosas técnicas biotecnológicas cada vez más precisas (Solórzano, 2011; Muñoz \& Johnston, 2013; Doan, Mason, Castoe, Sasa \& Parkinson, 2016). A la fecha de esta revisión se pueden considerar un total de 142 especies de serpientes en Costa Rica (Chaves, Bolaños, 
Rodríguez \& Matamoros, 2014), de las cuales sólo 73 especies (Apéndice 2) habitan en la GAM, según el extenso registro histórico de su distribución geográfica (Savage, 2002; Solórzano, 2004; Muñoz \& Johnston, 2013).

Finalmente, es importante aclarar que este trabajo implica dos suposiciones inevitables: que la presencia o ausencia de serpientes en cierto lugar responde únicamente a las condiciones de altitud y clima del mismo, como factores limitantes para la fisiología de las serpientes (Savage, 2002; Solórzano, 2004); y que las serpientes siguen habitando donde alguna vez fueron reportadas (Savage, 2002; Solórzano, 2004; Chaves et al., 2014).

Sin embargo, se sabe que esto no es necesariamente cierto y que, al igual que otros grupos faunísticos, algunas especies de serpientes son más tolerantes a la alteración del hábitat que otras, de manera que es muy probable que el urbanismo ya haya reducido el número de especies de serpientes en la GAM (Savage, 2002; Solórzano, 2004). Los estudios poblacionales de serpientes son muy escasos y se ocuparía uno para cada especie para aventurarse a dar un número más exacto a la realidad cotidiana (Savage, 2002; Solórzano, 2004; Chaves et al., 2014).

\section{Serpientes de jardín}

De las 73 especies de serpientes reportadas para la GAM, 21 tienen hábitos fosoriales, semifosoriales o de hojarasca (Apéndice 2), lo cual significa que habitan en túneles o escondidas debajo de hojas secas, troncos, piedras, macetas, adornos y objetos similares frecuentes en los jardines (Savage, 2002; Solórzano, 2004).

Las serpientes de jardín de la GAM son fáciles de reconocer (Fig. 2). La mayoría son del grosor de un dedo meñique y miden entre $15 \mathrm{~cm}$ y $50 \mathrm{~cm}$, con dos excepciones que pueden alcanzar los $80 \mathrm{~cm}$ (Savage, 2002; Solórzano, 2004). Suelen ser de colores poco llamativos como negro, pardo y pardo rojizo, tres especies tienen rayas longitudinales y seis especies poseen el vientre amarillo $u$ rojo (Savage, 2002; Solórzano, 2004).

Las Ilamadas "culebras de jardín", "serpientes hojarasqueras" o "culebras de tierra", son inofensivas a los humanos y nos generan beneficios al consumir invertebrados como: escorpiones, grillos, lombrices, arañas o cucarachas (Savage, 2002; Solórzano, 2004). También es importante mencionar que, aunque nueve especies son vistas en jardines con relativa frecuencia, la mayoría son consideradas "raras" y se les ve muy poco, probablemente por sus hábitos subterráneos y nocturnos (Savage, 2002; Solórzano, 2004).

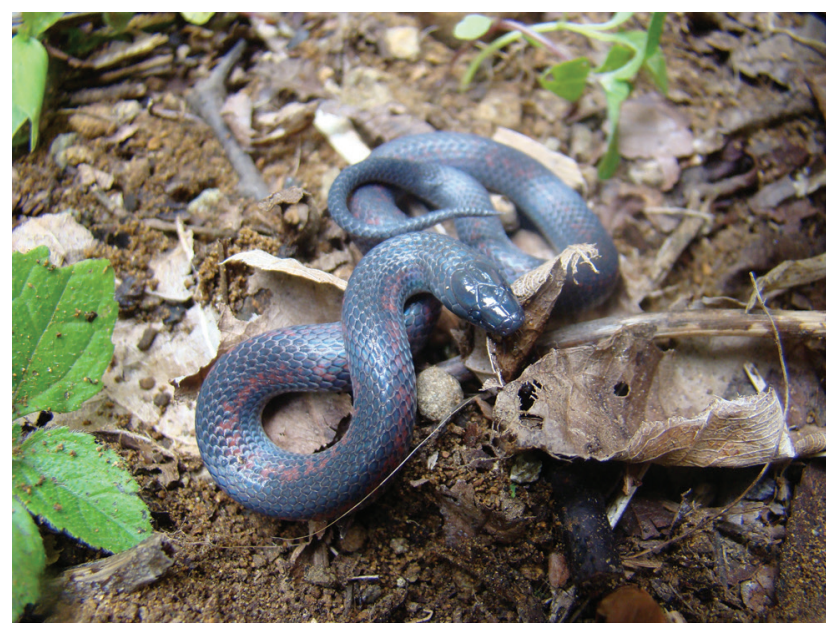

Fig. 2. Las serpientes de jardín habitan escondidas debajo de troncos, hojarasca y objetos similares, son del grosor de un dedo meñique y miden menos de $50 \mathrm{~cm}$ de largo. Fotografía de Laura Artavia.

\section{Serpientes de río}

En la GAM sólo se han reportado dos especies de serpientes semi-acuáticas o asociadas directamente a los cursos de agua (Apéndice 2) y considerando que se alimentan de animales acuáticos como camarones, cangrejos, ranas y peces; es probable que la contaminación de los ríos urbanos juegue un papel importante en su distribución actual (Whitfield et al., 2000; Savage, 2002; Solórzano, 2004; Barrientos, 2010).

Las serpientes de río de la GAM se reconocen porque habitan en el agua o muy cerca de ella (Fig. 3), una de

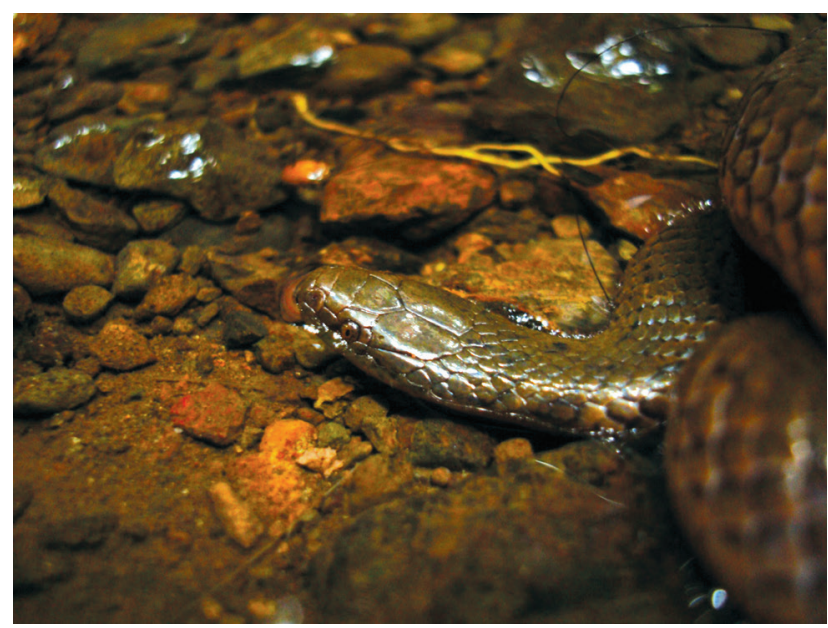

Fig. 3. En la GAM sólo se han reportado dos especies de serpientes semi-acuáticas y es probable que la contaminación de los ríos urbanos disminuya su distribución actual. Fotografía de Miguel Matarrita. 
las especies es color pardo grisáceo o café y la otra tiene rayas longitudinales en colores pardo y amarillo verdoso (Savage, 2002; Solórzano, 2004).

Al igual que las serpientes de jardín, las llamadas "culebras acuáticas" o "camaroneras" son inofensivas a los humanos, aunque su aspecto y tamaño es mucho más intimidante. Ambas especies son del grosor de un dedo índice y miden entre $70 \mathrm{~cm}$ y $100 \mathrm{~cm}$ (Savage, 2002; Solórzano, 2004).

\section{Serpientes de árbol}

Es posible que las serpientes arborícolas sean más reconocidas (y temidas) por la población que las serpientes de los dos grupos antes mencionados, aunque para la GAM únicamente se han reportado 16 especies (Apéndice 2) y sólo tres representan un verdadero peligro para las personas (Savage, 2002; Solórzano, 2004).

Reconocer a las serpientes arborícolas es relativamente sencillo considerando que por lo general evitan el contacto con el suelo y son del grosor de un dedo índice (Fig. 4). Sin embargo, su longitud varía considerablemente según la ecología de las especies y basándonos en eso, se podrían considerar dos subgrupos: diez serpientes arborícolas de la GAM son largas (entre $100 \mathrm{~cm}$ y $210 \mathrm{~cm}$ ), lo que les ha valido el nombre de "bejuquillas", "pajareras" o "falsa lora" cuando se ven durante el día, o"culebras ojos de gato" cuando se ven durante la noche (Savage, 2002; Solórzano, 2004; Muñoz \& Johnston, 2013). Las serpientes de este subgrupo son agresivas pero inofensivas, y suelen ser de colores muy apropiados para la vida en los árboles, como verde, café, café con machas o pardos amarillentos (Savage, 2002; Solórzano, 2004).

Las otras seis serpientes arborícolas de la GAM son medianas (entre $60 \mathrm{~cm}$ y $100 \mathrm{~cm}$ ) y presentan colores brillantes y llamativos como amarillo, verde, rojo, dorado, rosado, gris, blanco y negro, en infinidad de combinaciones (Savage, 2002; Solórzano, 2004). Las serpientes de este subgrupo son nocturnas, pacíficas y de andar lento: tres especies son completamente inofensivas y pasan sus noches cazando caracoles; pero las otras tres son venenosas y ocasionan accidentes principalmente cuando las personas las tocan por error mientras ellas descansan en algún arbusto (Savage, 2002; Solórzano, 2004).

Las tres serpientes arborícolas venenosas de la GAM (Fig. 5) pertenecen a la familia Viperidae y tienen nombres comunes célebres y temidos: la "lora", verde con pequeñas líneas claras a los lados del cuerpo; la "bocaracá", "oropel" o "toboba de pestañas", de brillantes colores y distintivas proyecciones en las escamas sobre los ojos; y la "toboba de árbol", verde con manchas negras,

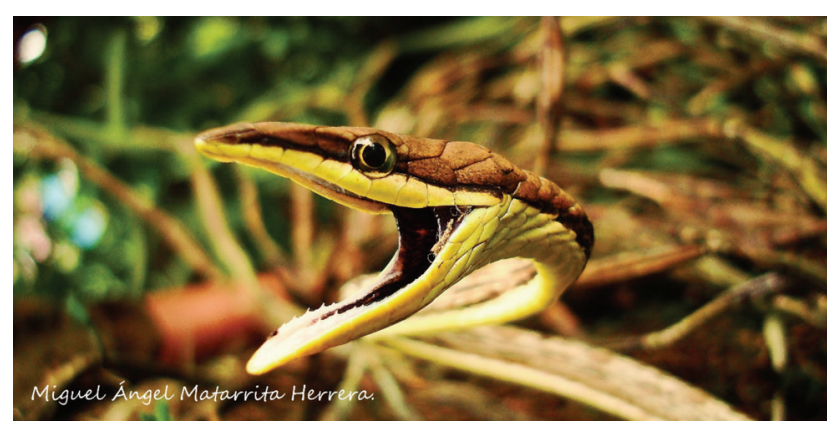

Fig. 4. La mayoría de las serpientes arborícolas de la GAM son largas (más de $100 \mathrm{~cm}$ ), delgadas y exhiben colores apropiados para la vida en los árboles, como verde, café, café con manchas o pardo. Fotografía de Miguel Matarrita.

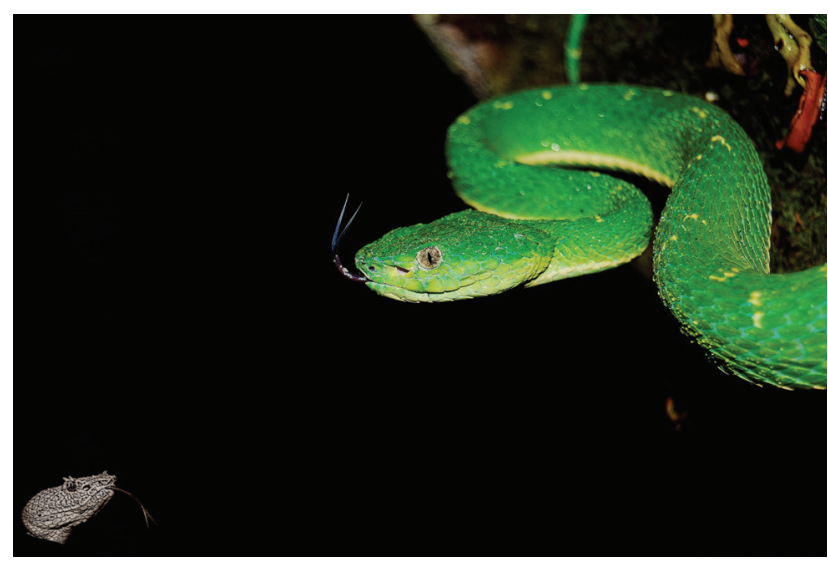

Fig. 5. Las tres serpientes arborícolas venenosas de la GAM son medianas (menos de $100 \mathrm{~cm}$ ) y presentan colores brillantes en infinidad de combinaciones. La "lora" es verde con pequeñas líneas claras a los lados del cuerpo. Fotografía de Miguel Matarrita.

aunque bastante difícil de encontrar (Savage, 2002; Solórzano, 2004).

\section{Serpientes terrestres}

Finalmente, de las 73 especies de serpientes reportadas para la GAM, 34 son especialistas en desplazarse por el suelo (algunas a gran velocidad) y alcanzan tamaños y grosores realmente intimidantes (Apéndice 2), aunque sólo ocho representan un verdadero peligro para las personas por su veneno (Savage, 2002; Solórzano, 2004). A continuación, y únicamente para efectos didácticos, me referiré a las 34 serpientes terrestres de la GAM dividiéndolas en cuatro subgrupos.

La serpiente más grande del país, la "boa" o "becker", compone por sí misma el primer subgrupo de serpientes 
terrestres de la GAM (familia Boidae), porque puede alcanzar hasta $450 \mathrm{~cm}$ de longitud y el grosor de un brazo (Fig. 6). Sin embargo, pese a lo que sugiere su gran tamaño, esta serpiente de hermosas manchas cafés no representa ningún riesgo para las personas adultas y lamentablemente, sus crías han ganado popularidad como mascotas exóticas (Arias-Ortega, Bonilla-Murillo \& Sasa, 2016). Las serpientes crecen con sus dueños hasta que éstos ya no pueden mantenerlas (por alimento y espacio) y terminan siendo liberadas en lugares inapropiados (Savage, 2002; Solórzano, 2004; Arias-Ortega et al., 2016).

Dentro del segundo subgrupo de serpientes terrestres de la GAM (20 especies) incluyo las Ilamadas "corredoras", "sabaneras", "guardacaminos", "ratoneras", "micas" y la "zopilota" (Apéndice 2), nombres que hacen referencia a su extraordinaria velocidad, hábitos alimenticios o aspecto físico (Savage, 2002; Solórzano, 2004) (Fig. 7). Todas alcanzan largos entre $100 \mathrm{~cm}$ y $300 \mathrm{~cm}$, grosores mayores a dos dedos índice y presentan colores como café, amarillo, verde, blanco y negro, a veces en combinaciones bicolor o

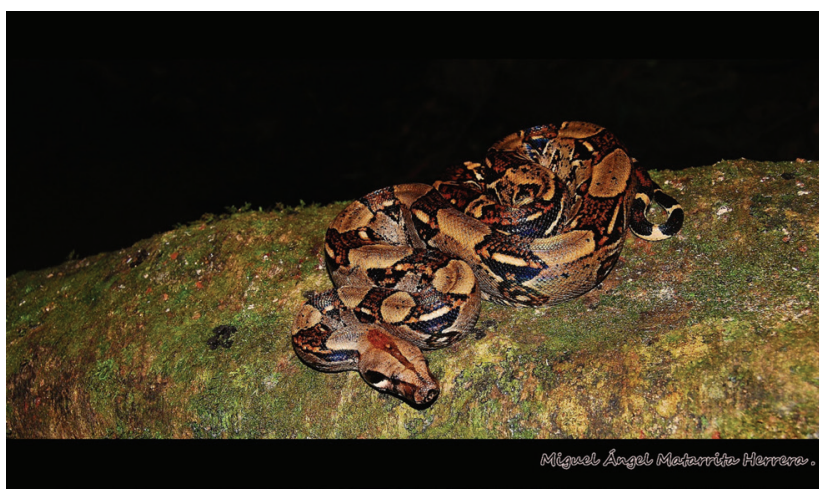

Fig. 6. La serpiente terrestre más grande del país es la "boa" o "becker", puede alcanzar hasta $450 \mathrm{~cm}$ de largo y el grosor de un brazo. Sus crías han ganado popularidad como mascotas exóticas. Fotografía de Miguel Matarrita.

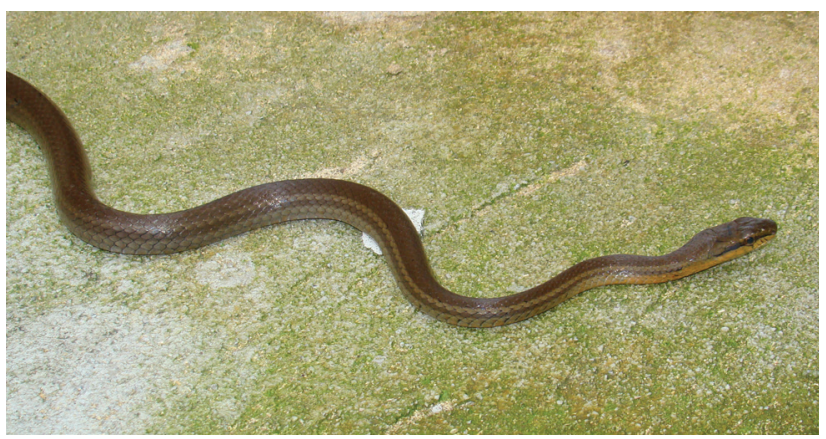

Fig. 7. Las serpientes terrestres de la GAM: "corredoras", "sabaneras", "guardacaminos", "ratoneras", "micas" o"zopilota", reciben nombres comunes en referencia a su velocidad, su alimento o apariencia. Fotografía de Michael Méndez. con rayas longitudinales (Savage, 2002; Solórzano, 2004). Valga mencionar que algunas, como la "mica tigre o pajarera", tienen gran habilidad para subir árboles y llamativas combinaciones de color en tonos verde, amarillo y negro (Savage, 2002; Solórzano, 2004).

Las serpientes terrestres del segundo subgrupo suelen estar activas durante el día y son agresivas, aunque no son peligrosas; de hecho, pueden beneficiar a los agricultores de la GAM al controlar plagas en los cultivos e incluso la "zopilota" (negra con vientre claro) se alimenta de serpientes venenosas. Lamentablemente, los agricultores matan a las "zopilotas" juveniles (cabeza negra, collar claro y cuerpo rojo) erróneamente convencidos de que son "víboras de sangre" venenosas (Savage, 2002; Solórzano, 2004).

En el tercer subgrupo de serpientes terrestres de la GAM coloco a las célebres "corales" $y$ "falsas corales", porque muchos no saben distinguirlas con certeza (Fig. 8). Se trata de ocho especies (Apéndice 2) cuya coloración presenta anillos de color rojo, amarillo, anaranjado, blanco y negro, en diferentes órdenes (Savage, 2002; Solórzano, 2004). Las tres especies de "corales" venenosas de la GAM (familia Elapidae) miden entre $70 \mathrm{~cm}$ y $110 \mathrm{~cm}$, tienen la cara color negro, ojos muy pequeños y presentan anillos completos de tres colores, en el orden rojo-amarillo-negro-amarillo (R-A-N-A); o anillos completos de dos colores, alternando negro con otro color como rojo, anaranjado o blanco (Savage, 2002; Solórzano, 2004).

En todo caso, la población de la GAM puede confiar en que las "corales" se caracterizan por tener un temperamento pasivo y tímido, lo cual, sumado a la poca capacidad de apertura de su boca, resulta en que las probabilidades de sufrir un accidente por mordedura de serpiente coral son muy bajas (Savage, 2002; Solórzano, 2004).

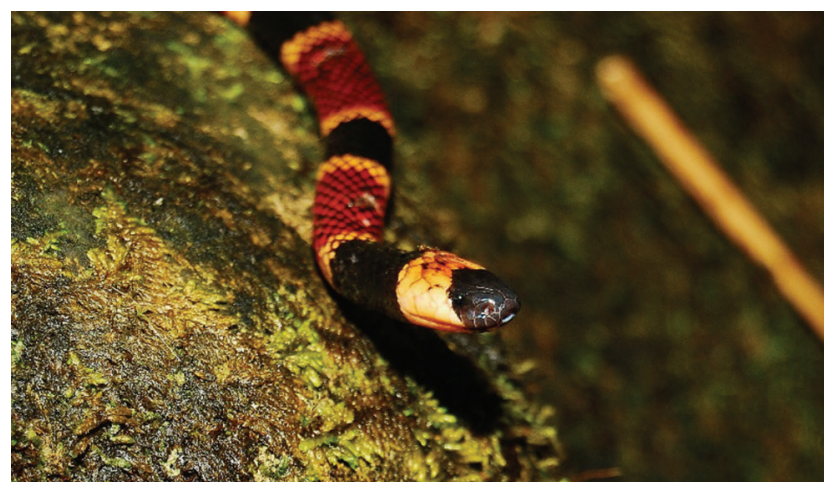

Fig. 8. Las tres especies de corales venenosas de la GAM miden menos de $100 \mathrm{~cm}$, tienen ojos pequeños y presentan anillos completos de tres colores (R-A-N-A) o de dos colores (N-A-N-A o N-R-N-R). Fotografía de Miguel Matarrita. 


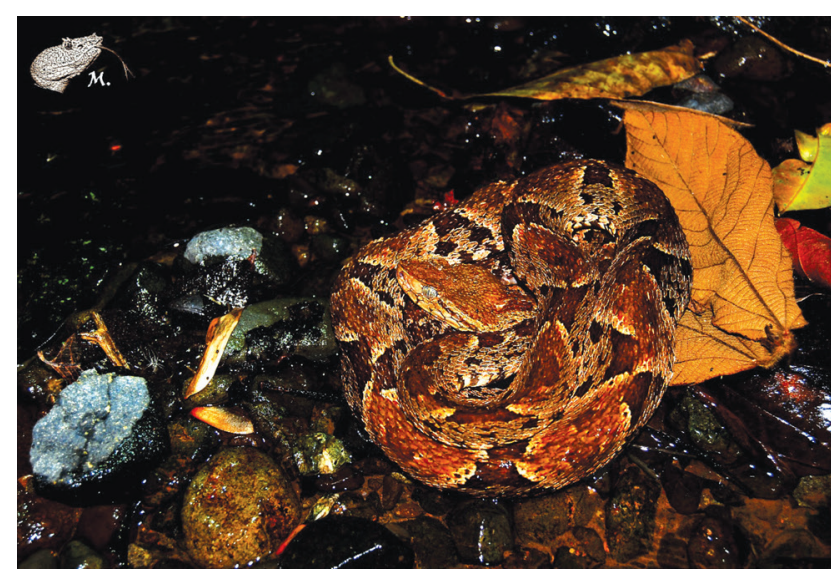

Fig. 9. Las serpientes venenosas más peligrosas de la GAM (como la terciopelo) suelen estar camufladas en el suelo y presentan manchas romboidales en combinaciones bicolor, con tonos de café, gris, pardo, blanco y negro. Fotografía de Miguel Matarrita.

El último subgrupo de serpientes terrestres de la GAM incluye cinco especies venenosas (Apéndice 2) cuyos nombres generan temor, aunque muchos nunca las hayan visto (Prokop et al., 2009; Torkar, 2015; Pinheiro et al., 2016). Las especies de este subgrupo (familia Viperidae) alcanzan distintos tamaños, son malas trepadoras y presentan manchas romboidales en hermosas combinaciones bicolores, con tonos de café, gris, pardo, blanco y negro. Su camuflaje natural les permite permanecer quietas en el suelo esperando a su presa; y esto también genera accidentes, cuando las personas no las ven y se acercan demasiado (Savage, 2002; Solórzano, 2004).

Pertenecen a este subgrupo: la "mano de piedra" y la "toboba", que difícilmente sobrepasan los $90 \mathrm{~cm}$ de largo; la "cascabel", de hasta $170 \mathrm{~cm}$ incluyendo su inconfundible cascabel; y la "terciopelo" (Fig. 9), que puede alcanzar los $230 \mathrm{~cm}$ y posiblemente sea la serpiente que le da origen a la injusta mala fama de todas las demás especies (Savage, 2002; Solórzano, 2004).

\section{CONCLUSIÓN}

Según el registro histórico de su distribución geográfica (Savage, 2002; Solórzano, 2004), en la GAM costarricense (Apéndice 1) habitan 73 especies de serpientes (Apéndice 2), de las cuales sólo 11 representan un verdadero peligro para la salud humana en caso de acercarse demasiado, con o sin intención (Savage, 2002; Solórzano, 2004).

El temor a las serpientes suele fundamentarse en mitos e historias ficticias (Prokop et al., 2009), pues ciertamente la mayoría son inofensivas y podemos coexistir con ellas en paz, por el bien de los ecosistemas y la biodiversidad (Solórzano, 2004; Nonga \& Haruna, 2015).

Al encontrar una serpiente en la GAM es necesario recordar que no hay nada que temer, y evaluar las distintas formas en las que se puede actuar, tal y como lo hacemos al encontrar un ave golpeada o un gato. En la mayoría de los casos una escoba bastará para ahuyentar a la serpiente, pero también es posible llamar a los bomberos o pedir ayuda a algún biólogo de su comunidad si el animal es grande o de una especie de cuidado (Solórzano, 2004).

Finalmente, lo invito a que busque lugares especializados para que pueda conocer más sobre las serpientes costarricenses, pues la educación ambiental hará que usted sustituya sus miedos, por genuino interés (Torkar, 2015; Pinheiro et al., 2016).

\section{AGRADECIMIENTOS}

Agradezco a Gabriela Pérez, Néstor Quesada, Julián Monge, Juan Abarca, Daniela Vargas, Zaidett Barrientos y Alejandro Solórzano por sus sugerencias para mejorar el manuscrito. También a Laura Artavia, Michael Méndez y Miguel Matarrita por facilitar las fotografías para ilustrar el artículo.

\section{REFERENCIAS}

Arias-Ortega, J., Bonilla-Murillo, F., \& Sasa, M. (2016). Desarrollo de la herpetocultura en Costa Rica: Situación actual de herpetarios y manejo ex situ de reptiles y anfibios. Revista de Ciencias Ambientales, 50(1), 1-23. doi: 10.15359/rca.50-1.1

Barrientos, Z. (2010). Problemática de los ciclos biogeoquímicos, hidrológico y de nutrientes en la meseta central de Costa Rica. Posgrado y Sociedad, 10(1), 23-37.

Barrientos, Z., \& Monge-Nájera, J. (2010). Especies introducidas en la meseta central de Costa Rica. Biocenosis, 23(2), 32-37.

Chaves, G., Bolaños, F., Rodríguez, J. E., \& Matamoros, Y. (Eds.) (2014). Actualización de las listas rojas nacionales de Costa Rica: Anfibios y reptiles; utilizando los lineamientos de las listas rojas de la UICN (Informe Final). San José, Costa Rica: Conservation Breeding Specialist Group (CBSG Mesoamérica).

Doan, T. M., Mason, A. J., Castoe, T. A., Sasa, M., \& Parkinson, C. L. (2016). A cryptic palm-pitviper species (Squamata: Viperidae: Bothriechis) from the Costa Rican highlands, with notes on the variation within B. nigroviridis. Zootaxa, 4138(2), 271-290. doi: 10.11646/zootaxa.4138.2.3 
Leenders, T. (2001). A guide to amphibians and reptiles of Costa Rica. San José, Costa Rica: Zona Tropical Publications.

McConnell, G. J. (2013). A field guide to the snakes of Costa Rica. Frankfurt-Main, Alemania: Edition Chimaira.

Ministerio de Planificación Nacional y Política Económica (MIDEPLAN) (2014). Plan Nacional de Desarrollo 20152018: Alberto Cañas Escalante. San José, Costa Rica: MIDEPLAN.

Muñoz, F., \& Johnston, R. D. (2013). Anfibios y reptiles de Costa Rica: Guía de bolsillo en inglés y español. Nueva York, Estados Unidos: Cornell University Press.

Nonga, H. E., \& Haruna, A. (2015). Assessment of human-snake interaction and its outcomes in Monduli District, northern Tanzania. Tanzania Journal of Health Research, 17(1), 1-12. doi: 10.4314/thrb.v17i1.9

Pinheiro, L. T., Mota, J. F., \& Borges-Nojosa, D. M. (2016). Formal education, previous interaction and perception influence the attitudes of people toward the conservation of snakes in a large urban center of northeastern Brazil. Journal of ethnobiology and ethnomedicine, 12(25), 1-8. Doi: 10.1186/s13002-016-0096-9

Prokop, P., Özel, M., \& Uşak, M. (2009). Cross-cultural comparison of students attitudes toward snakes. Society and animals, 17, 224-240. doi: 10.1163/156853-009-X-445398
Instituto Nacional de Vivienda y Urbanismo (INVU) (2011). Plan de ordenamiento territorial de la Gran Área Metropolitana (2011-2030). San José, Costa Rica: INVU.

Savage, J. M. (2002). Amphibians and reptiles of Costa Rica: A herpetofauna between two continents, between two seas. Chicago, Estados Unidos: University of Chicago Press.

Sistema Nacional de Áreas de Conservación (SINAC) (2014). V Informe Nacional al Convenio sobre Diversidad Biológica, Costa Rica. San José, Costa Rica: SINAC.

Solórzano, A. (2004). Serpientes de Costa Rica: Distribución, taxonomía e historia natural. Heredia, Costa Rica: Instituto Nacional de Biodiversidad (INBio).

Solórzano, A. (2011). Variación de color de la serpiente marina Pelamis platura (Serpentes: Elapidae) en el Golfo Dulce, Puntarenas, Costa Rica. Cuadernos de Investigación UNED, 3(1), 89-96.

Torkar, G. (2015). Pre-service teachers' fear of snakes, conservation attitudes, and likelihood of incorporating animals into the future science curriculum. Journal of Baltic Science Education, 14(3), 401-410.

Whitfield, J., Scott, D. E., Ryan, T. J., Buhlmann, K. A., Tuberville, T. D., Metts, B. S., Greene, J. L., Mills, T., Leiden, Y., Poppy, S., \& Winne, C. T. (2000). The global decline of reptiles, déja vu amphibians. BioScience, 50(8), 653-666. 


\section{APÉNDICE 1}

Lista de los cantones y distritos que forman parte del Gran Área Metropolitana (GAM) de Costa Rica (INVU, 2011; MIDEPLAN, 2014).

\begin{tabular}{|c|c|c|}
\hline Provincia & Cantón & Distritos \\
\hline \multirow[t]{13}{*}{ San José } & San José & $\begin{array}{l}\text { Carmen, Catedral, Hatillo, Hospital, Mata Redonda, Merced, Pavas, San Francisco de Dos Ríos, } \\
\text { San Sebastián, Uruca y Zapote }\end{array}$ \\
\hline & Escazú & Escazú, San Antonio y San Rafael \\
\hline & Desamparados & $\begin{array}{l}\text { Damas, Desamparados, Gravilias, Patarrá, Rosario, San Antonio, San Juan de Dios, San Miguel, } \\
\text { San Rafael Abajo y San Rafael Arriba }\end{array}$ \\
\hline & Aserrí & Aserrí y Salitrillos \\
\hline & Mora & Colón \\
\hline & Goicoechea & $\begin{array}{l}\text { Calle Blancos, Guadalupe, Ipís, Mata de Plátano (Carmen), Purral, Rancho Redondo } \\
\text { y San Francisco }\end{array}$ \\
\hline & Santa Ana & Brasil, Piedades, Pozos, Santa Ana, Salitral y Uruca \\
\hline & Alajuelita & Alajuelita, Concepción, San Antonio, San Felipe y San Josecito \\
\hline & Vásquez de Coronado & Cascajal, Dulce Nombre de Jesús, Patalillo, San Isidro y San Rafael \\
\hline & Tibás & Anselmo Llorente, Cinco Esquinas, Colima, León XIII y San Juan \\
\hline & Moravia & La Trinidad, San Jerónimo y San Vicente \\
\hline & Montes de Oca & Mercedes, Sabanilla, San Pedro y San Rafael \\
\hline & Curridabat & Curridabat, Granadilla, Sánchez y Tirrases \\
\hline \multirow[t]{3}{*}{ Alajuela } & Alajuela & $\begin{array}{l}\text { Alajuela, Carrizal, Desamparados, Garita, Guácima, Río Segundo, Sabanilla, San Antonio, San } \\
\text { Isidro, San José, San Rafael, Tambor y Turrúcares }\end{array}$ \\
\hline & Atenas & Atenas, Concepción, Jesús y Mercedes \\
\hline & Poás & Carrillos, Sabana Redonda, San Juan, San Pedro y San Rafael \\
\hline \multirow[t]{6}{*}{ Cartago } & Cartago & $\begin{array}{l}\text { Aguacaliente (San Francisco), Carmen, Dulce Nombre, Guadalupe (Arenilla), Llano Grande, } \\
\text { Occidental, Oriental, Quebradillas, San Nicolás y Tierra Blanca }\end{array}$ \\
\hline & Paraíso & Cachí, Orosi, Paraíso y Santiago \\
\hline & La Unión & Concepción, Dulce Nombre, Río Azul, San Diego, San Juan, San Rafael, San Ramón y Tres Ríos \\
\hline & Alvarado & Capellades, Cervantes y Pacayas \\
\hline & Oreamuno & Cipreses, Cot, San Rafael, Santa Rosa y Potrero Cerrado \\
\hline & El Guarco & San Isidro, Tejar y Tobosi \\
\hline \multirow[t]{9}{*}{ Heredia } & Heredia & Heredia, Mercedes, San Francisco y Ulloa \\
\hline & Barva & Barva, San José de la Montaña, San Pablo, San Pedro, San Roque y Santa Lucía \\
\hline & Santo Domingo & Pará, Paracito, San Miguel Sur, San Vicente, Santa Rosa, Santo Domingo, Santo Tomás y Tures \\
\hline & Santa Bárbara & Jesús, Purabá, San Juan Abajo, San Pedro, Santa Bárbara y Santo Domingo \\
\hline & San Rafael & Ángeles, Concepción, San Josecito, San Rafael y Santiago \\
\hline & San Isidro & Concepción, San Isidro, San José y San Francisco \\
\hline & Belén & Asunción, Ribera y San Antonio \\
\hline & Flores & Barrantes, Llorente y San Joaquín \\
\hline & San Pablo & San Pablo \\
\hline
\end{tabular}




\section{APÉNDICE 2}

Lista de las 73 especies de serpientes que habitan en la GAM, Costa Rica, según su distribución geográfica histórica (Savage, 2002; Solórzano, 2004; Muñoz \& Johnston, 2013).

\begin{tabular}{|c|c|c|c|c|c|}
\hline Familia & Nombre científico & Hábitos & $\begin{array}{l}\text { Rango Altitud } \\
\text { (m.s.n.m.) }\end{array}$ & Frecuencia & Nombre común \\
\hline Anomalepididae & Helminthophis frontalis & Fosorial-Hojarasca & $900-1200$ & Rara & Culebra de jardín \\
\hline Boidae & Boa imperator & Terrestre & $0-1500$ & Común & Boa o Becker \\
\hline \multirow[t]{28}{*}{ Colubridae } & Chironius exoletus & Terrestre & $500-1600$ & Rara & Mica verde \\
\hline & Chironius grandisquamis & Terrestre & $0-1600$ & Común & Mica negra \\
\hline & Dendrophidion clarkii & Terrestre & $100-1000$ & Rara & Corredora café \\
\hline & Dendrophidion paucicarinatum & Terrestre & $1000-1800$ & Común & Corredora verde \\
\hline & Dendrophidion percarinatum & Terrestre & $0-1200$ & Común & Corredora café \\
\hline & Dendrophidion rufiterminorum & Terrestre & $100-1000$ & Rara & Corredora café \\
\hline & Drymarchon melanurus & Terrestre & $0-1600$ & Común & Sabanera café \\
\hline & Drymobius margaritiferus & Terrestre & $0-1500$ & Común & Sabanera verde \\
\hline & Lampropeltis micropholis & Terrestre & $650-2500$ & Rara & Coral falsa \\
\hline & Leptodrymus pulcherrimus & Terrestre & $0-2000$ & Rara & Corredora \\
\hline & Leptophis depressirostris & Arborícola & $0-1300$ & Común & Lora falsa \\
\hline & Leptophis mexicanus & Arborícola & $0-1300$ & Común & Lora falsa \\
\hline & Leptophis nebulosus & Arborícola & $20-1600$ & Común & Lora falsa \\
\hline & Masticophis mentovarius & Terrestre & $0-1400$ & Común & Chilillo café \\
\hline & Mastigodryas melanolomus & Terrestre & $0-1700$ & Común & Corredora café \\
\hline & Oxybelis aeneus & Arborícola & $0-1400$ & Común & Bejuquilla café \\
\hline & Oxybelis brevirostris & Arborícola & $10-1200$ & Común & Bejuquilla verde \\
\hline & Oxybelis fulgidus & Arborícola & $0-1600$ & Común & Bejuquilla verde \\
\hline & Phrynonax poecilonotus & Arborícola & $0-1300$ & Común & Mica pajarera \\
\hline & Scolecophis atrocinctus & Terrestre & $0-1500$ & Rara & Coral falsa \\
\hline & Senticolis triaspis & Terrestre & $0-1500$ & Común & Ratonera café \\
\hline & Spilotes pullatus & Terrestre & $0-1500$ & Común & Mica tigre o real \\
\hline & Stenorrhina degenhardtii & Semifosorial & $5-1400$ & Común & Escorpionera \\
\hline & Stenorrhina freminvillii & Semifosorial & $10-1500$ & Común & Escorpionera \\
\hline & Tantilla armillata & Semifosorial & $0-1400$ & Rara & Hojarasquera \\
\hline & Tantilla ruficeps & Semifosorial & $0-1600$ & Rara & Hojarasquera \\
\hline & Tantilla schistosa & Semifosorial & $50-1500$ & Rara & Hojarasquera \\
\hline & Tantilla supracincta & Semifosorial & $0-900$ & Rara & Hojarasquera \\
\hline \multirow[t]{13}{*}{ Dipsadidae } & Clelia clelia & Terrestre & $0-900$ & Común & Zopilota \\
\hline & Clelia scytalina-equatoriana & Terrestre & $400-2000$ & Común & Zopilota \\
\hline & Coniophanes fissidens & Terrestre & $0-1150$ & Común & Guardacaminos \\
\hline & Enulius flavitorques & Fosorial-Hojarasca & $0-1300$ & Común & Culebra de tierra \\
\hline & Erythrolamprus bizona & Terrestre & $0-1600$ & Común & Coral falsa \\
\hline & Geophis brachycephalus & Semifosorial & $10-2100$ & Común & Culebra de tierra \\
\hline & Geophis godmani & Semifosorial & $1300-2200$ & Común & Culebra de tierra \\
\hline & Geophis hoffmanni & Semifosorial & $20-1200$ & Común & Culebra de tierra \\
\hline & Geophis zeledoni & Semifosorial & $1700-2100$ & Rara & Culebra de tierra \\
\hline & Hydromorphus concolor & Semiacuática & $0-1500$ & Común & Camaronera \\
\hline & Imantodes cenchoa & Arborícola & $0-1600$ & Común & Bejuquilla café \\
\hline & Imantodes gemmistratus & Arborícola & $0-1400$ & Común & Bejuquilla café \\
\hline & Leptodeira rhombifera & Terrestre & $0-1400$ & Común & Ojos de gato \\
\hline
\end{tabular}




\begin{tabular}{|c|c|c|c|c|c|}
\hline Familia & Nombre científico & Hábitos & $\begin{array}{l}\text { Rango Altitud } \\
\text { (m.s.n.m.) }\end{array}$ & Frecuencia & Nombre común \\
\hline & Leptodeira septentrionalis & Arborícola & $0-1500$ & Común & Ojos de gato \\
\hline & Liophis epinephalus & Terrestre & $0-2100$ & Rara & Culebra de fuego \\
\hline & Ninia maculata & Semifosorial & $0-1800$ & Común & Hojarasquera \\
\hline & Ninia psephota & Semifosorial & $1000-2000$ & Rara & Hojarasquera \\
\hline & Pliocercus euryzonus & Terrestre & $0-1500$ & Rara & Coral falsa \\
\hline & Rhadinaea calligaster & Semifosorial & $1200-2500$ & Rara & Hojarasquera \\
\hline & Rhadinaea decorata & Semifosorial & $0-1000$ & Común & Hojarasquera \\
\hline & Rhadinaea pulveriventris & Semifosorial & $1300-1600$ & Rara & Hojarasquera \\
\hline & Rhadinella godmani & Semifosorial & $1300-2600$ & Rara & Hojarasquera \\
\hline & Rhadinella serperaster & Semifosorial & $1200-2200$ & Común & Hojarasquera \\
\hline & Sibon anthracops & Arborícola & $0-900$ & Común & Caracolera \\
\hline & Sibon dimidiatus & Arborícola & $25-1650$ & Rara & Caracolera \\
\hline & Sibon nebulatus & Arborícola & $0-1200$ & Común & Caracolera \\
\hline & Trimetopon gracile & Semifosorial & $1250-2250$ & Rara & Hojarasquera \\
\hline & Trimetopon pliolepis & Semifosorial & $60-1550$ & Rara & Hojarasquera \\
\hline & Urotheca decipiens & Terrestre & $0-1600$ & Rara & Hojarasquera \\
\hline & Urotheca guentheri & Terrestre & $0-1700$ & Rara & Hojarasquera \\
\hline \multirow[t]{3}{*}{ Elapidae } & Micrurus alleni & Terrestre & $0-1600$ & Común & Coral verdadera \\
\hline & Micrurus mipartitus & Terrestre & $0-1600$ & Rara & Coral gargantilla \\
\hline & Micrurus nigrocinctus & Terrestre & $0-1500$ & Común & Coral verdadera \\
\hline Natricidae & Thamnophis proximus & Semiacuática & $0-1700$ & Común & Camaronera \\
\hline Sibynophiidae & Scaphiodontophis venustissimus & Terrestre & $0-1250$ & Rara & Coral falsa \\
\hline \multirow[t]{8}{*}{ Viperidae } & Atropoides mexicanus & Terrestre & $50-1600$ & Común & Mano de piedra \\
\hline & Atropoides picadoi & Terrestre & $300-2000$ & Rara & Mano de piedra \\
\hline & Bothriechis lateralis & Arborícola & $450-2500$ & Común & Lora verdadera \\
\hline & Bothriechis nigroviridis & Arborícola & $1350-3000$ & Rara & Toboba \\
\hline & Bothriechis schlegelii & Arborícola & 0-1500 & Común & Bocaracá \\
\hline & Bothrops asper & Terrestre & $0-1500$ & Común & Terciopelo \\
\hline & Cerrophidion sasai & Terrestre & $1400-3000$ & Común & Toboba \\
\hline & Crotalus simus & Terrestre & $0-1800$ & Común & Cascabel \\
\hline
\end{tabular}

